\title{
Potentials of sustainable bio Diesel fuels to achieve mobility
}

Dr. Ralf Türck, Tecosol GmbH

Zum Zeitpunkt der Drucklegung lagen diese Unterlagen noch nicht vor.

Wir bitten um Verständnis. 